\title{
La interacción de las actividades orales y escritas en la enseñanza de la argumentación
}

\author{
Joaquim Dolz
}

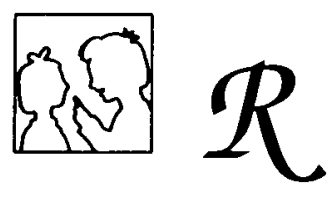

Partiendo de la creación de situaciones de comunicación variadas, el autor plantea la enseñanza de la argumentación oral a partir de la observación, la manipulación y la reflexión metalingüística de las características linguiisticas y discursivas de este tipo de texto. Las investigaciones llevadas a cabo en el marco de la escuela primaria ponen en evidencia que existe un efecto de retorno entre el monólogo argumentativo escrito y el monólogo argumentativo oral.

El presente artículo se propone explorar las posibilidades, el interés y los límites de la articulación de las actividades orales y escritas en la enseñanza de la argumentación. Tradicionalmente, el desarrollo de la argumentación oral ha sido considerado como un requisito necesario para abordar la argumentación escrita pero raramente han sido estudiadas de manera rigurosa las diferencias y las interdependencias complejas entre ambas modalidades. Para poder discutir la relación lengua oral/lengua escrita desde un punto de vista linguiístico y didáctico, nos parece indispensable clarificar dicha oposición en función del tipo de interacción verbal adoptado (diálogo, discusión, debate, monólogo) y de la situación de producción propuesta. La interdependencia será analizada en dos direcciones distintas. Por una parte, examinaremos de manera crítica hasta qué punto la realización de actividades orales sobre algunas estrategias argumentativas pueden servir de base y/o integrarse en una secuencia de enseñanza sobre el monólogo argumentativo escrito. Por otra parte, sugeriremos ciertas actividades de escritura de monólogos argumentativos susceptibles de ejercer un efecto de retorno sobre la argumentación oral.

\section{LAS MODALIDADES INSTRUMENTALES ORAL Y ESCRITA}

El desarrollo de la expresión oral, como el de la expresión escrita, constituye uno de los grandes objetivos de la escuela obligatoria. Sin embargo, en el caso de la enseñanza de la lengua materna, el aprendizaje de la lectura, de la escritura, de la grámatica y de la ortografía, continuan siendo puntos mucho más sensibles. La renovación pedagógica de los últimos años ha supuesto una clarificación en relación a la 
enseñanza/aprendizaje de la composición escrita, entendida como producción de diferentes tipos de textos, sin que exista hasta ahora un trabajo equivalente sobre la producción oral. Como denuncian sociólogos, lingüistas y formadores (Wirthner, Martin y Perrenoud, 1991), la enseñanza formal de la lengua oral y de su uso en diferentes situaciones de comunicación ocupa actualmente un lugar limitado. Los medios didácticos y las indicaciones metodológicas son bastante escasas. La formación del profesorado presenta lagunas importantes. Para comprender las dificultades de la legitimación escolar de la lengua oral podemos evocar al menos tres razones.

En primer lugar existe la convicción, por parte de numerosos enseñantes, de que la expresión oral se adquiere y se desarrolla de manera informal fuera de la escuela. El papel del enseñante en ese ámbito resulta secundario. Le basta con crear situaciones de comunicación oral, corregir individualmente algunos errores y valorizar el trabajo libre y espontáneo de los alumnos. La enseñanza formal, el trabajo escolar sistemático, queda reservado para estudiar el código, las normas y el funcionamiento del lenguaje escrito.

En segundo lugar, quienes consideran que hay que escolarizar la expresión oral como instrumento para asegurar la confianza y el dominio de diversas situaciones de interacción social, especialmente para los alumnos menos favorizados, facilitando de esta manera su éxito en los estudios y en la vida profesional, disponen de pocos elementos teóricos y prácticos susceptibles de mejorar la expresión de sus alumnos. Subsisten numerosas preguntas: ¿qué prácticas sociales tomar como referencia? ¿cuáles son los contenidos de la enseñanza de la lengua oral? ¿qué actividades de observacion, de manipulación, de producción, de evaluación deben llevarse a cabo?

En tercer lugar, la distinción entre las formas de realización del lenguaje oral y del escrito es portadora de numerosas confusiones.

La primera confusión consiste en presentar la lengua escrita como un simple sistema sustitutivo de la lengua oral (natural) o la expresión escrita como una simple transposición de la expresión oral. En realidad, durante siglos, la lengua oral fue abordada por los lingüistas sobre la base de datos de la lengua escrita y raramente a partir del análisis de corpus orales. Pero, como analiza pertinentemente Mosteirin (1991), la escritura como sistema de notación del lenguaje oral, presenta un carácter incompleto e inexacto.

La segunda confusión consiste, una vez reconocida la especificidad de la lengua oral, en no ver más que las divergencias. Por ejemplo, algunos autores presentan la sintaxis de la lengua hablada y de la lengua escrita como una oposición radical y sobrevaloran las diferencias a todos los niveles de realización linguística (ver las críticas a los «mitos separadores» de Blanche-Benveniste y Jeanjean, 1987). También es común, en ciertos medios ideologizados, atribuir juicios de valor a la dicotomía oral/escrito en función de los registros o niveles de habla: la lengua hablada es considerada como pobre, vulgar y mal estructurada mientras que la lengua escrita constituye la base de toda norma de corrección. Esta simplificación ignora las múltiples posibilidades de escribir en una variedad popular o familiar y de hablar en un registro culto o académico. La especificidad de la modalidad oral de un discurso no es necesariamente más clara para aquellos que adoptan una perspectiva enunciativa y textual. Por ejemplo, hay quienes confunden las características enunciativas y estructurales del discurso interactivo dialogal con las de su realización oral y olvidan que el diálogo escrito puede presentar características similares (cf. Adam, 1993).

La riqueza y la complejidad de la expresión oral es subrayada por los lingüistas (cf. Roulet, 1991) cuando afirman que no exite un sólo tipo de lengua oral sino una pluralidad de orales en función de las distintas situaciones de comunicación, regidos por normas distintas. Curiosamente, aquellos que reconocen la diversidad y la complejidad de la lengua oral relativizan su oposición con la lengua escrita. Sin negar las 
diferencias comunicativas y lingüísticas entre lengua oral y lengua escrita, se dibuja actualmente una nueva tendencia que trata de evitar presentarlos como dos ámbitos completamente separados. Para Roulet, la observación y el análisis de las estructuras de la lengua oral, más alla de las diferencias morfológicas y situacionales, permite renovar el estudio de las estructuras de la lengua y del discurso en general.

En el marco de la elaboración de un modelo del funcionamiento de los discursos (Bronckart y cols, 1985), los grandes tipos de discurso (narración, relato conversacional, discurso teórico y discurso interactivo) no son considerados como típicamente orales o escritos. En las formulaciones más recientes del mismo modelo, Bronckart (1992) presenta el discurso como la actualización de una acción verbal y opone fundamentalemente CONTAR (narraciones, relatos, etc.) y EXPONER (discurso informativo, argumentativo, etc.). En el primer caso, el enunciador se distancia de los acontecimientos que cuenta y de la situación de interacción social. En el segundo caso, presenta estados, nociones o acontecimientos en el mismo mundo de los protagonistas de la interacción. Los discursos de uno y otro orden pueden presentar un grado de IMPLICACION O AUTONOMIA más o menos pronunciado: la monogestión o poligestión son una de las consecuencias fundamentales. Según el grado de IMPLICACION-AUTONOMIA, Bronckart distingue la NARRACION ( «Érase una vez una ratita que se llamaba Paquita...», «El año 1492, Aragón y Castilla...») y el RELATO INTERACTIVO ( La semana pasada, paseaba por la calle y me encontré con uno de tus amigos. Cuando me acerqué...»), el Discurso TEOrico («Este libro analiza cinco conceptos que han dominado todos los debates semióticos...») y el Discurso INTERACTIVO (- Señor presidente ¿Podría darnos su opinión sobre las últimas declaraciones de Alfonso Guerra? - No veo qué relación tiene mi opinión sobre las declaraciones del señor Guerra con el tema de nuestra entrevista.). Las características esenciales de los cuatro tipos de discurso que acabamos de presentar no dependen de su realización oral o escrita. Por ejemplo, el diálogo del último ejemplo, es probablemente la transcripción de un fragmento de una entrevista oral pero podría ser perfectamente parte de un diálogo escrito, aparecido en una de las novelas de Vázquez Montalbán.

Toda acción verbal (y en consecuencia todo género discursivo) es susceptible de adoptar una modalidad oral o escrita (Dolz, Pasquier y Bronckart, 1993). Por ejemplo, podemos relatar un hecho histórico oralmente y por escrito; entrevistar a un personaje oralmente o por escrito y difundir esa misma entrevista por la radio o por la prensa escrita. La presentación de un monólogo expositivo, argumentativo y/o explicativo puede realizarse oralmente en forma de conferencia magistral o por escrito en forma de editorial de prensa, artículo enciclopédico, etc. Sin embargo, el uso y las convenciones hacen preferible ciertas modalidades de realización orales o escritas en algunas situaciones de comunicación.

Actualmente, la influencia respectiva de la realización oral o escrita tiende a ser estudiada a través de géneros discursivos producidos en situaciones de comunicación semejantes. Por ejemplo, las investigaciones de los miembros del equipo de investigación de Bronckart confirman que la materialización oral o escrita de una narración (de Weck, 1991) o de un monólogo argumentativo (Rosat, 1991) se manifiesta fundamentalmente en la utilización de ciertas unidades lingüísticas específicas (en el caso de las narraciones, ciertas categorías de anáforas distinguen la narración oral de la escrita y, en el caso de los monólogos argumentativos ciertas categorías de organizadores textuales). Los resultados de estas investigaciones coinciden con los análisis realizados de los prototipos textuales de Adam (1993). Para este autor, la organización secuencial de un prototipo textual (descriptivo, narrativo, explicativo, argumentativo o dialogal) no depende de su realización oral o escrita.

En cuanto a la enseñanza de la lengua oral, durante los años 70 , proliferaron las «técnicas de expresión» que ofrecían un panorama bastante confuso de habilida- 
des de todo orden, con un cierto predominio de los aspectos socio-afectivos y relacionales (atreverse a hablar, poner la tensión emocional al servicio del habla, respetar al interlocutor, jugar con las palabras, dramatizar situaciones). En ese terreno, hay que señalar un avance considerable en cuanto a la diversidad de situaciones de comunicación tomadas en consideración y a sus características contextuales específicas (Grisay y Delanshere, 1989): gravación de mensajes en el contestador automático, improvisación de conversaciones, dramatización de situaciones diversas, descripción de itinerarios con o sin la ayuda de un mapa, conferencia, etc.. En particular, la creación de situaciones funcionales variadas destinadas a provocar el uso del habla ha mejorado notablemente gracias al análisis de prácticas sociales de referencia y a la reflexión sobre los mecanismos que intervienen en la producción (Bain, en este mismo número). Steffen (1991) habla de tres tipos de situaciones para desarrollar el lenguaje oral: - realización de un proyecto de grupo (por ejemplo, la representación de un espectáculo para una fiesta); - participación en redes de comunicación del centro escolar en relación con el exterior (radio escolar) o destinadas a mejorar la gestión interna (creación de un consejo o de una asamblea de clase); - secuencias de comunicación simuladas (juegos de comunicación, juegos de roles y los momentos de discusión).

Sin negar el interés pedagógico de las innovaciones introducidas durante los últimos años sobre la práctica de la lengua oral, la creación de situaciones de comunicación variadas aparece actualmente como una condición necesaria, pero no suficiente, para la enseñanza de la lengua oral. Un mínimo de actividades de observación, de manipulación y de reflexión metalingüística sobre las principales características de una práctica discursiva específica parecen igualmente necesarias (Bain, op. cit.; Rosat, 1993). La selección de contenidos de la lengua oral para la enseñanza y la organización de éstos en secuencias de enseñanza constituyen uno de los retos de la didáctica de la lengua (ver los artículos de Bain y de Besson y Canelas-Trevisi, en este mismo número).

\section{LAS CAPACIDADES ARGUMENTATIVAS ORALES Y ESCRITAS}

Los estudios de psicología muestran la existencia de un desfase entre el desarrollo de las capacidades argumentativas orales y escritas (Bereiter y Scardamalia, 1982). Dicho desfase se explica fundamentalmente por la dificultad de los alumnos a tratar la dimensión dialógica de la argumentación escrita. Un niño es capaz relativamente pronto de defender en una conversación su punto de vista sobre un tema que le interese (François, 1980). En el diálogo argumentativo oral, la presencia de dos interlocutores «cara a cara» aparece como fundamental para facilitar la toma en consideración del punto de vista del otro. El niño se adapta con mayor facilidad y de manera inmediata a la posición del contrario, mientras que, en un monólogo argumentativo oral o escrito (Rosat, 1991), debe realizar un esfuerzo mucho mayor para identificar la finalidad y el destinatario del discurso. La diferenciación de su punto de vista y el del adversario continua siendo problemática. El niño adopta normalmente un punto de vista estático e introduce difícilmente modificaciones a la posición inicial.

Producir un monólogo argumentativo oral o escrito supone: anticipar globalmente la posición del destinatario, justificar y apoyar su punto de vista con un conjunto de argumentos, rechazar los posibles argumentos contrarios, planificar la sucesión de los argumentos y su articulación, negociar una posición aceptable para todos. Según Brassart (1989), los niños no consiguen contruir argumentaciones elaboradas, articulando argumentos y contra-argumentos, hasta los 12-13 años. 
Los estudios sobre la adquisición «natural» de la argumentación escrita (Schneuwly, 1988) muestran un orden regular en la puesta en funcionamiento de las operaciones centrales del discurso argumentativo, la justificación y la negociación (cf. Golder, 1992): hacia los 10-11 años, los niños son capaces de apoyar con una serie de argumentos una opinión; hacia los 13-14 años, comienzan a modalizar su texto y a distanciarse; a los 16 años, introducen sistemáticamente argumentos y estrategias que suponen la negociación de su posición con un punto de vista distinto (ya sea el del destinatario o el de un posible adversario).

Corier y Golder (1991) distinguen cinco niveles de organización estructural del monólogo argumentativo. En un primer nivel, el niño no toma una posición explícita en la controversia. En un segundo nivel, el niño formula de manera explícita su posición pero sin un razonamiento para apoyarla. En un tercer nivel, el niño introduce un argumento para apoyar su posición. En los niveles cuatro y cinco, la argumentación se complica por la presencia de varios argumentos que son progresivamente puestos en relación entre ellos. A esos cinco niveles, presentados de manera esquemática, habría que añadir otro nivel implicando la introducción de argumentos de negociación.

En una investigación reciente (Dolz, en prensa a), hemos mostrado que una de las razones de la adquisición tardía del monólogo argumentativo es la ausencia de enseñanza sistemática en la escuela. La enseñanza precoz de la argumentación no comporta dificultades que no se puedan superar a condición de:

- observar y tomar en cuenta las capacidades argumentativas de los alumnos antes de la enseñanza;

- seleccionar y operacionalizar contenidos de enseñanza apropiados, relativos al discurso argumentativo;

- establecer una progresión coherente;

- proponer actividades pedagógicas interesantes para los alumnos.

\section{LA INTERACCION ORAL/ESCRITO EN LA ENSEÑANZA/APRENDIZAJE DE LA ARGUMENTACION}

El especialista en didáctica confrontado a la elaboración de secuencias didácticas destinadas a mejorar la expresión del alumno no puede dejar de interrogarse sobre las relaciones complejas existentes entre las modalidades oral y escrita.

1. ¿Hasta qué punto el desarrollo de la modalidad oral constituye un requisito indispensable para el aprendizaje de la modalidad escrita? ¿Podemos dar una respuesta general a esta cuestión con un mínimo de validez para todo tipo de discurso y para las diferentes capacidades verbales que supone su producción?

2. ¿En qué medida es conveniente separar, integrar o poner en relación las actividades orales y escritas en una secuencia de enseñanza? ¿En qué situaciones los ejercicios de producción oral contribuyen a la preparación del alumno hacia la producción escrita? En el sentido contrario, ¿podemos esperar que el ejercicio de la producción escrita contribuya a mejorar la expresión oral de los alumnos?

Crear diferentes situaciones de argumentación orales y escritas en todas las etapas de la escolaridad

Generalmente se admite que cierto grado de dominio del funcionamiento del lenguaje oral constituye la base sobre la que se efectua la adquisición del lenguaje escrito. Podemos admitir que la adquisición del discurso oral interactivo constituye 
un prerequisito para la adquisición de los aspectos dialógicos de todo discurso, incluídos los monólogos escritos (narraciones, argumentaciones, explicaciones, etc.). El diálogo oral aparece como la forma natural y externa de un mecanismo enunciativo mucho más complejo analizado por Bajtin en términos de polifonía (presencia de diferentes voces en una sola intervención) y dialogismo (toma en consideración de la dimensión interactiva de la circulación discursiva).

Las investigaciones actuales muestran, sin embargo, que es difícil abordar las relaciones entre la expresión oral y escrita de manera general sin tomar en consideración el género de discurso y las condiciones de producción particulares. Desde ese punto de vista, cada vez aparece como más reduccionista la hipótesis de una gradación-sucesión entre los géneros discursivos según la cual el discurso interactivo oral serviría de base para el desarrollo de la narración y el aprendizaje de esta última serviría a su vez de base para el desarrollo de la argumentación y de la explicación; formas discursivas consideradas relativamente tardías ponen en duda esta concepción reduccionista de la progresión de los aprendizajes (cf. Dolz, Pasquier y Bronckart, 1993).

Cada discurso (y probablemente cada género discursivo) presenta elementos característicos que exigen un aprendizaje específico. Por ejemplo, el monólogo argumentativo se distingue de otros discursos por la situación de interacción particular que se establece entre el argumentador, un posible adversario y el destinatario así como por la presencia de numerosas estrategias expresivas y propiedades lingüísticas específicas. Resulta pues bastante improbable que los alumnos desarrollen las capacidades específicas de la argumentación, trabajando únicamente sobre las características típicas de la narración. Pero resulta también bastante improbable que los alumnos desarrollen ciertas capacidades específicas del monólogo argumentativo si solamente practican el diálogo argumentativo.

En relación a la planificación del currículum escolar, coincidimos con la posición de Bain (1991) cuando considera más prudente «renunciar a toda una planificación general del currículum imponiendo globalmente una serie de etapas de aprendizaje para el conjunto de escolaridad o durante el año académico». En efecto, una secuencialización fija del currículum del tipo: primero, el debate oral; en segundo lugar, la conferencia oral y en tercer lugar, el monólogo argumentativo escrito, resulta totalmente artificial y difícilmente justificable en función de los conocimientos que disponemos sobre el funcionamiento de los diferentes géneros argumentativos orales y escritos. Todo parece indicar que hay que ejercer una diversidad de géneros argumentativos orales y escritos a lo largo de toda la escolaridad.

En algunos casos, por ejemplo, se pretende poner en relación los progresos del aprendizaje de la argumentación oral y de la argumentación escrita y en realidad se comparan dos géneros discursivos distintos, la discusión y el monólogo argumentativo. Rosat (1991) muestra que los alumnos entre 10 y 14 años encuentran grosso modo los mismos problemas cuando tiene que producir (en una situación similar, a partir del mismo objetivo y del mismo referente) un monólogo argumentativo oral o escrito. En ambos casos, la producción del monólogo argumentativo exige del alumno un distanciamiento respecto al objeto de la controversia y una fuerte estructuración interna de carácter distinto al diálogo argumentaivo. Rosat muestra cómo la progresión de la estructuración de las dos primeras modalidades se produce por un uso cada vez más diferenciado de ciertas unidades linguiísticas.

La escuela debería proponer una diversidad de actividades orales metódicas como:

- la discusión improvisada sobre un tema polémico (en favor o en contra de los animales domésticos, de los deberes escolares, de la igualdad de la mujer, etc.); 
- la entrevista con el representante de una posición determinada para comprender sus argumentos, ponerlos en duda o refutarlos (entrevista con una autoridad escolar, con una feminista, con un adulto partidario de retrasar la edad de obtención del permiso de conducir, etc.);

- el debate de confrontación organizado entre dos personas o dos grupos equilibrados representando posiciones distintas sobre un problema de actualidad;

- la exposición en público o la conferencia sobre un tema controvertido (por ejemplo, la prohibición de la circulación en el centro de las grandes ciudades) para defender con argumentos y contra-argumentos un punto de vista determinado.

Las formas de interacción y los medios para obtener la adhesión del destinatario difieren considerablemente entre los cuatro géneros orales que acabo de presentar. Como difieren también entre los géneros argumentativos escritos, ya sea por el lugar social donde se producen (texto publicitario, editorial de prensa, artículo de opinión, carta del lector al diario, petición, panfleto, texto judicial, etc.), ya sea por la funciones sociales del enunciador y del destinatario (reclamación del ciudadano, respuesta de la autoridad en la correspondencia oficial, administrativa y jurídica), ya sea por el carácter mismo de los valores objeto de la controversia (deliberación sobre decisiones políticas, establecimiento de normas morales, justificación de una sanción). Antes de establecer la relación entre las diferentes situaciones de argumentación orales y escritas en la enseñanza, hay que subrayar la importancia de la diversificación en todas las etapas de la escolaridad.

Algunos problemas de los alumnos cuando abordan la argumentación son específicos a las diversas situaciones que acabamos de evocar. En consecuencia, sólo podemos esperar que mejoren sus capacidades argumentativas si los familiarizamos con esas situaciones. Como afirma Bain (1991), la práctica de la discusión oral difícilmente puede considerarse como propedéutica para el aprendizaje de ciertas marcas lingüísticas de conexión, de cohesión y de modalización propias de los géneros argumentativos escritos que acabamos de presentar. Los diálogos argumentativos son a menudo de una complejidad enorme. García (1990) afirma que, en el aprendizaje de la discusión y del debate contradictorio, las formas de interacción entre los participantes y la legitimación personal de cada uno de ellos revisten tanta importancia como los contenidos. En esas situaciones, «ocupar el terreno» es tan importante como la construcción progresiva del objeto de la argumentación y las estrategias para convencer al interlocutor. A veces, cuando mayor es el acuerdo entre los participantes sobre los contenidos, mayor es el desacuerdo y la violencia que se manifiesta en la relación. En esas situaciones, se mezclan inextricablemente la lógica de los argumentos evocados y las estrategias discursivas ligadas a la dinámica del grupo (tomar la palabra, ceder la palabra, monopolizar la palabra, respetar la circulación de los intercambios, tener en cuenta o ignorar los argumentos del otro, desplazar la problemática, bloquear la discusión con una intervención agresiva, etc.). Desde el punto de vista relacional, un argumento en una discusión oral debe satisfacer las condiciones siguientes para ser eficaz:

a) ser escuchado por los otros participantes;

b) dar lugar a una interacción (el silencio es una forma de rechazar los argu-. mentos considerados como no pertinentes);

c) suscitar la aprobación de otros participantes (estrategias de alianza);

d) dejar al adversario sin posibilidad de réplica;

e) obligar al interlocutor a cambiar de terreno.

Enfin, sería ingenuo imaginar que la gestión de esas situaciones de comunicación es menos problemática que la de los monólogos argumentativos orales y escritos. 
En el marco de una investigación realizada en las escuelas primarias ginebrinas para evaluar los efectos sobre la escritura de una enseñanza sistemática e intensiva de la argumentación, hemos elaborado y experimentado una serie de secuencias didácticas sobre ese tipo de discurso (Dolz, 1993; Cotteron 1993; Dolz y Pasquier 1993 a y 1993 b; Dolz, en prensa a y b).

Las secuencias didácticas han sido concebidas como un conjunto ordenado de talleres destinados a mejorar las capacidades argumentativas de los alumnos. Básicamente, en los talleres se recurre a cuatro tipos de actividades:

1.- contrastar diversas situaciones de argumentación y textos argumentativos para favorecer la toma de conciencia de las relaciones entre los parámetros de las situaciones de interacción y las estrategias argumentativas utilizadas;

2.- elaborar diferentes tipos de argumentos o de contra-argumentos y organizarlos en el texto;

3.- practicar situaciones orales y escritas de colaboración con el fin de facilitar la transición progresiva de la simple oposición a los argumentos del adversario, muy frecuente en los alumnos de esas edades, hacia las estrategias de negociación que suponen una coordinación de puntos de vista para llegar a un compromiso;

4.- ejercer por escrito (tareas simplificadas de producción de textos argumentativos) ciertas estrategias lingüísticas características de la argumentación:

- las formas personales de la y 2a persona para expresar la posición del argumentador (desde mi punto de vista) o implicar al destinatario en su razonamiento ( $V d s$ estarán de acuerdo conmigo);

- los organizadores textuales lógico-argumentativos para asegurar la conexión entre los argumentos (causales, de oposición, de conclusión, etc.);

- las modalizaciones para indicar la fuerza de un argumento (estoy seguro que, probablemente, etc.) y las restricciones de una conclusión; autoridad;

- el estilo directo, indirecto e indirecto libre para introducir citaciones de

- los verbos que introducen una opinión valorándola (demostrar, afirmar) o degradándola (pretender);

- las expresiones destinadas a formular objeciones (dudo que...);

- las locuciones concesivas (sin duda tiene usted razón pero...; aunque sea indispensable debemos...).

Las secuencias didácticas se organizan según los principios siguientes. En primer lugar, el enseñante propone y discute con sus alumnos un proyecto de escritura. Los alumnos participan en la definición del proyecto, conocen los objetivos y participan en su planificación. El proyecto de escritura se concretiza con la puesta en práctica de una situación de comunicación. Los alumnos producen entonces un primer texto que permite al profesor identificar ciertas dificultades relativas a la escritura de textos argumentativos. Ese primer texto permite igualmente elegir los contenidos de enseñanza y las actividades pedagógicas que serán realizadas en los distintos talleres. En segundo lugar, los alumnos realizan las diversas actividades seleccionadas: debates orales; análisis y observación de textos, ejercicios de producción simplicada; juegos, ejercicios de vocabulario, ejercicios sobre las unidades lingüísticas y sobre las expresiones características de la argumentación. Finalmente, gracias a lo que han aprendido, revisan y re-escriben el primer texto o escriben un nuevo texto argumentativo. Esta última actividad permite al alumno tomar conciencia de los progresos realizados desde el comienzo de la secuencia didáctica. 
La experimentación, realizada en colaboración con los enseñantes de varios centros escolares, nos ha permitido aislar ciertos aspectos relativos a la interacción oral/escrito que vamos a resumir brevemente.

1. Todas las secuencias didácticas experimentadas partían de una situación argumentativa definida previamente y perseguían objetivos relativos a un monólogo argumentativo escrito. El hecho de seleccionar objetivos de trabajo relativos a una situación argumentativa bien delimitada constituye una de las condiciones para que los alumnos den sentido a las actividades realizadas y perciban su carácter finalizado. Perseguir simultáneamente objetivos relativos a distintas situaciones de comunicación ya sean orales o escritas en una misma secuencia de enseñanza puede dispersar la atención y crear confusiones en los alumnos de esas edades.

2. Aunque las secuencias didácticas estén orientadas a mejorar la escritura de textos argumentativos, durante su realización, las actividades orales y escritas se interpenetran constantemente y de forma casi inevitable. En primer lugar, los juegos de simulación en los que los alumnos «dramatizan» diferentes personajes (el juez, el abogado, el fiscal y los jurados populares) permiten que el alumno analice y comprenda rápidamente ciertos parámetros de las situaciones de argumentación. En segundo lugar, para no alargar en exceso la secuencia didáctica y para evitar un exceso de cansancio en los alumnos, ciertas actividades se realizan oralmente. Por ejemplo, antes de participar individualemente por escrito a un debate propuesto por una revista infantil sobre los animales domésticos, los alumnos elaboran oralmente los argumentos que los padres podrían evocar contra la presencia de un gato o de un perro en casa y luego discuten colectivamente cómo podrían rebatir cada uno de los argumentos. En tercer lugar, todos los talleres de trabajo comportan una fase de puesta en común en la que se debaten oralmente las distintas soluciones a los problemas surgidos. Una técnica practicada corrientemente con los alumnos es el dictado al adulto; el dictado al adulto invierte la dinámica del dictado tradicional: los alummnos oralizan el texto que quieren escribir para que el maestro lo copie en el encerado; durante el dictado, el maestro pregunta constantemente a los alumnos sobre el trabajo que están realizando. Por otra parte, la oralización en público de los textos escritos producidos a lo largo de toda la secuencia didáctica resulta una práctica importante para que los alumnos tomen conciencia de la gran variedad de estrategias argumentativas utilizadas. La heterogeneidad de las respuestas dadas en clase se convierte así en una fuente colectiva de aprendizajes. La integración de actividades orales sobre algunas situaciones y estrategias argumentativas en una secuencia sobre la argumentación escrita aparece, en los casos citados y por diversas razones, como inevitable. Enseñar la expresión oral y escrita de manera íntimamente unida puede representar una gran riqueza, a condición de que se tomen las precauciones necesarias para evitar confusiones entre las distintas situaciones de comunicación.

3. Las actividades pedagógicas de las secuencias didácticas experimentadas son fundamentalmente actividades escritas. Al final de la secuencia didáctica, los alumnos producen un texto escrito. En una de las situaciones de simulación, por ejemplo, los alumnos interpretan el papel de un concejal de un ayuntamiento que escribe un informe destinado a sus colegas para defender la realización de un proyecto.

Una vez terminada la secuencia didáctica, hemos querido observar si el trabajo realizado por los alumnos en esa situación ejerce un efecto de retorno sobre la argumentación oral. Para ello, les hemos pedido que imaginen los cambios que deberían introducir en sus informes escritos si tuvieran que defender el mismo proyecto oralmente en una junta del ayuntamiento. Los alumnos reflexionan entonces individual y colectivamente sobre las modificaciones lingüísticas que deben introducir en el texto escrito, si desean utilizarlo como apoyo para su exposición oral en grupo. Para facilitar la transición a la situación oral, cuando toman el papel del orador que dirige 
su palabra al público, se les aconseja que no lean su texto y que presten atención a la entonación, al ritmo y a las pausas para asegurar la inteligibilidad de la exposición y su carácter persuasivo.

Una vez que han tomado conciencia de la diferencia entre las dos situaciones y de algunos aspectos específicos de la exposición oral, los alumnos utilizan selectivamente lo que aprendieron sobre la argumentación en los talleres de escritura. Todo parece indicar entonces que el trabajo y la reflexión sobre el monólogo argumentativo escrito ejerce un efecto de retorno sobre el monólogo argumentativo oral. Las formas concretas de este tipo de regulación deberían ser objeto de nuevas investigaciones.

\section{Referencias}

ADAM, J.-M. (1993) Les textes: types et prototypes, Natham Université, París

CotTERoN, J. (1993), Sensibilisation au texte d'opinion: «Entre chien et chat...débat», en Cabiers du Service du Français, 28, DIP, Ginebra

BAIN, D. (1991) «L'argumentation orale prépare-t-elle au texte argumentatif écrit», en WIRTHNER, M.; Martin, D. y Perrenoud, Ph. (Eds) Parole étouffée, parole libérée. Fondements et limites d'une pédagogie de l'oral, Neuchâtel-París, Delachaux y Niestlé, pp. 159-164

BerEITER, C. y SCARDAMALIA, M (1987) The psychology of uritten composition. Hillsdale, Erlbaum

BLANCHE-BeNveniste, C. y JEANJEAN, C. (1986) Le français parlé, édition et transcription, París, DidierErudition

BRASSART, D.-G. (1989) «Pourquoi et comment analyser et représenter le texte argumentatif (écrit)?», en Recherches, 9, pp. 123-164

Bronckart, J.-P.; Bain, D.; SChneuwly, B.; Davaud, C. y Pasquier, A. (1985) Le fonctionnement des discours, Neuchâtel-París, Delachaux y Niestlé

BRONCKART, J.-P. (1992) «El discurso como acción. Por un nuevo paradigma psicolinguístico», en Anuario de Psicología, 54, pp. 3-48

CORIER, P. y GOLDER, C. (1991) La production de textes argumentatifs: Etude développementale des structures d'étayage (documento policopiado)

DE WECK, G. (1991) La cobésion dans les textes d'enfants, Neuchâtel-París, Delachaux y Niestlé

Dolz, J. (1993) "La argumentación", en Cuadernos de Pedagogía, Monográfico Leer y escribir, 216, pP. 6870

DOIZ, J. (en prensa, a) «Learning argumentative capacities: a study of the effects of a systematic and intensive teaching of argumentative discourse en 11-12 year old children», en Argumentation.

Dolz, J. (en prensa, b) «Produire des textes pour mieux les comprendre. Etude de l'interaction écriturelecture à propos de l'enseignement du discours argumentatif», en Actes du Colloque de Lille sur l'interaction lecture-écriture, 1993

DOIZ, J. y PASQUIER, A. (1993a) «Argumenter... pour convaincre: initiation aux textes argumentatifs», en Cabiers du Service du Français, 31, DIP, Ginebra

Dolz, J. y PASQUIER, A. (1993b) “Mon avis, je l'écris': initiation aux textes d'opinion», en Cahiers du Service du Français, 35, DIP, Ginebra

DOlz, J.; PASQuier, A. y BronCKarT, J.-P. (1993, en prensa) «La adquisición de los discursos: ¿competencia emergente o aprendizaje de diversas capacidades verbales?", en Actas de las jornadas sobre la enseñanza de las lenguas de la EOI, Castellón de la Plana

François, F. (1980) «Dialogue, discussion et argumentation au début de la scolarité», en Pratiques, 28, PP. 83-94

GARCla, Cl. (1980) «Argumenter à l'oral: de la discussion au débat», en Pratiques, 28, pp. 95-124

Golder, C. (1992) «Argumenter: De la justification à la négociation», en Arcbives de Psychologie, 60, 232 , PP. 3-24

Grisay, A. y DELANSHERE, G. (1989) 30 situations de communication à l'oral, París, Labor

KeBrat-ORECCHIONI, C. (1990) Les interactions verbales 1 , París, Colin

LAFONTAINE, D. (1985) «Rapport oral/écrit: oú est la différence?», en Enjeux, 8, 46-60

Marchand, F. (1991) «Quelques aperçus sur l'enseignement de l'oral dans l'école héritée de Jules Ferry", en Repères, 3, 7-16

MOSTERIN, ). (1991) La ortografía fonémica del español, Madrid, Alianza

Rosat, M.-C. (1991) «A propos de la réalisation orale et écrite d'un texte argumentatif», en Etudes de Linguistique Appliquée, 87, Pp. 37-50

ROSAT, M.-C. (1993) «Réflechir pour la classe sur la diversité des oraux», en Le français aujourd"bui, 101, pp. $70-76$

Roulet, E. (1991) "La pédagogie de l'oral en question(s)», en Wirthner, M.; Martin, D. y Perrenoud, Ph. (Eds) Parole étouffée, parole libérée. Fondements et limites d'une pédagogie de l'oral, Neuchâtel-París, Delachaux y Niestlé, pp. 41-54 
SCHNEUWLY, B. (1988) Le langage érit chez l'enfant, Neuchâtel-París, Delachaux y Niestlé

SCHNEUWLY, B. y DOLZ, J. (1989) "La planification langagière chez l'enfant», en Revue suisse de psychologie pure et appliquée, $46(1,2)$, pp. 55-64

STEFFEN, N. (1991) «La communication orale à l'école», en Wirthner, M.; Martin, D. y Perrenouyd , Ph. (Eds) Parole étouffée, parole libérée. Fondements et limites d'une pédagogie de l'oral, Neuchâtel-París, Delachaux y Niestlé, pp. 127-148

WIRTHNer, M.; MARTIN, D. y Perrenoud, Ph. (Eds) (1991) Parole étouffée, parole libérée. Fondements et limites d’une pédagogie de l'oral, Delachaux \& Niestlé

\section{La interacción de las actividades orales y escritas en la enseñanza de la argumentación Joaquim Dolz CL\&E, 1994, 23, pp. 17-27}

Resumen: El presente artículo se ha propuesto explorar las posibilidades, el interés y los límites de la articulación de las actividades orales y escritas en la enseñanza de la argumentación. Para poder discutir la relación lengua oral/lengua escrita desde un punto de vista linguístico y didáctico, nos ha parecido indispensable clarificar dicha oposición en función del tipo de interacción verbal adoptado y de la situación de producción propuesta. La interdependencia entre ambas es analizada en dos direcciones distintas: examinando hasta qué punto la realización de actividades orales sobre algunas estrategias argumentativas pueden servir de base y/o integrarse en una secuencia de enseñanza sobre el monólogo argumentativo escrito; sugiriendo ciertas actividades de escritura de monólogos argumentativos susceptibles de ejercer un efecto de retorno sobre la argumentación oral.

Datos sobre el autor: Joaquim Dolz trabaja en la unidad de Didáctica de las lenguas de la Facultad de Psicología y Ciencias de la Educación de la Universidad de Ginebra, así como en el Servicio del francés del Departamento de la Instrucción Pública de esa misma ciudad, encargándose, entre otras cosas, de la elaboración de medios de enseñanza y de la formación del profesorado.

Dirección: Universidad de Ginebra, Facultad de Psicología y Ciencias de la Educación, Unidad de Didáctica de las lenguas, 9, Rte de Drize, CH - 1227 Ginebra (Suiza).

C PERMISOS PARA CITAR O REPRODUCIR EN OTRAS FUENTES: Se pueden citar libremente hasta 500 palabras. Para reproducir una porción de texto mayor, figuras o ilustraciones, se deberá pedir permiso por escrito a la revista, especificando el uso al que se destina el texto. En todos los casos, se deberá citar el copyright de $C L \& E$. En el caso de artículos o textos que hayan sido a su vez reproducidos en $C L \& E$ los interesados deberán dirigirse tanto a los detentadores del copyright original como a $C L \& E$, en el caso de que se quiera hacer uso de la traducción. FOTOCOPIAS: Para todo lo relacionado con el uso mediante fotocopia del material de esta revista, deberán dirigirse a: CEDRO, C/ José Marañón, 10, 3. ${ }^{\circ}$ Izda. Tel. 59415 75. Fax 4453567 